\title{
A Class of Explicitly Solvable Vehicle Motion Problems
}

\author{
J.M. Selig
}

\begin{abstract}
A small but interesting result of Brockett is extended to the Euclidean group $S E(3)$ and is illustrated by several examples. The result concerns the explicit solution of an optimal control problem on Lie groups, where the control belongs to a Lie triple system in the Lie algebra. The extension allows for an objective function based on an indefinite quadratic form.

Applying the result requires explicit knowledge of the Lie triple systems of the Lie algebra $s e(3)$. Hence, a complete classification of the Lie triple systems of this Lie algebra is derived.

Examples are considered for optimal trajectories in 3 cases. The first case concerns cars moving in the plane. The second looks at motions that rigidly follow the Bishop frame to a space curve. The final example does not have a particular name as it does not seem to have been studied before.

The appendix gives a brief introduction to Screw theory. This is essentially the study of the Lie algebra $s e(3)$.
\end{abstract}

Index Terms-Vehicles, Lie triple systems, optimal control.

\section{INTRODUCTION}

$\mathbf{I}$ T IS extremely rare to find an optimal control problem which has explicit solutions. Although the result presented here is rather technical it can be applied to the motion of cars and to Bishop motions. Bishop motions have been suggested as possible motions for serial robot arms to follow. The optimal curves for car motion can be used to interpolate between different positions of the car. In the final section these applications are studied together with a new type of rigid-body motion which appears as a result of the analysis but does not seem to have been considered previously.

This work was inspired by a short conference paper by Brockett, [3]. In that article explicit solutions are given for an optimal control problem on a Lie group under the condition that controls are restricted to a particular type of subset of the group's Lie algebra. Brockett called the structure required on the Lie algebra a $\mathbb{Z}_{2}$-grading. In [9] it was pointed out that Brockett's $\mathbb{Z}_{2}$-graded Lie algebra's were identical to the older concept of a Lie triple system. Rather, the grade 1 subspace is a Lie triple systems; the grade zero subspace is a subalgebra. There is yet another name for this structure in the literature: a Cartan algebra, [13]. The details of this structure are discussed in section IV below.

In the next section we begin with a discussion of the representation of conjugate variables. This provides the basic result for the extension of Brockett's theorem in Section III. It also serves to introduce the notation used.

In Section III an alternative proof of Brockett's theorem is offered, based on geometric control theory as outlined in [8]

J.M. Selig, is with the School of Engineering, London South Bank University, London SE1 0AA, U.K. e-mail: (seligjm@1sbu.ac.uk).

Manuscript received July 1, 2014; revised December 22, 2014. or [14]. Two small changes to the original are also made. In the original work Brockett considers right-invariant systems on Lie groups, here left-invariant systems are studied. Also here the possible performance measures or objective functions used in the original have been slightly extended. This allow the extension of the method to the Lie group $S E(3)$ which has no bi-invariant positive definite metric. The proof involves a dualisation of the Lie triple system or Cartan splitting, which does not seem to have been considered before.

Having demonstrated the importance of the Lie triple systems, the following section gives a complete classification of the Lie triple systems of $s e(3)$, the Lie algebra of the group of proper rigid body displacements.

Section V, contains an exposition of the three examples mentioned above; the car, the Bishop motion and a novel type of motion. An appendix gives a brief introduction to Screw theory; the Lie algebra $s e(3)$.

\section{Conjugate Variables}

In the following an element of a Lie algebra $\mathcal{L}$ will be denoted by a vector of the form $\mathrm{s}$. However, it will also be useful to consider the elements of the Lie algebra as square matrices. In this case the Lie algebra element will be written as $S$. The exact representation of the algebra used is not too important here, but for definiteness it may be assumed that the adjoint representation of the Lie algebra is being used. That is, $S=\operatorname{ad}(\mathbf{s})$ and hence the Lie bracket is given by, $\operatorname{ad}(\mathbf{s}) \mathbf{z}=S \mathbf{z}=[\mathbf{s}, \mathbf{z}]$.

The dual of the Lie algebra will be denoted $\mathcal{L}^{*}$ and dual vectors will be written $\mathbf{p}$. In classical language the components of the dual vector would be called conjugate variables. The evaluation map of a dual vector $\mathbf{p}$ on a vector in the Lie algebra can be written as the product, $\mathbf{p}^{T} \mathbf{s}$.

Given a basis of the Lie algebra, $\left\{\mathbf{s}_{1}, \ldots, \mathbf{s}_{n}\right\}$, where $n$ is the dimension of the Lie algebra, it is possible to find a dual basis for the dual space, $\left\{\mathbf{p}_{1}, \ldots, \mathbf{p}_{n}\right\}$. That is,

$$
\mathbf{p}_{i}^{T} \mathbf{s}_{j}= \begin{cases}1, & \text { if } i=j, \\ 0, & \text { if } i \neq j .\end{cases}
$$

The coadjoint representation of the Lie algebra on its dual can be written as $\operatorname{ad}^{*}(\mathbf{s}) \mathbf{p}=\{\mathbf{s}, \mathbf{p}\}$. This curly bracket notation is due to Arnol'd, [1, Appendix 2]. This representation is defined by the relation,

$$
\{\mathbf{s}, \mathbf{p}\}^{T} \mathbf{z}=\mathbf{p}^{T}[\mathbf{z}, \mathbf{s}] .
$$

In terms of matrices it can be seen that, $\operatorname{ad}^{*}(\mathbf{s})=-\operatorname{ad}(\mathbf{s})^{T}$, that is the coadjoint representation is the negative transpose of the adjoint representation. 
Now consider a linear map $Q: \mathcal{L} \longrightarrow \mathcal{L}^{*}$. Such a map can be represented, with respect to the dual coordinate system by a symmetric matrix, which will also be denoted $Q$. Now suppose this map satisfies the following relation,

$$
Q \operatorname{ad}(\mathbf{s})-\operatorname{ad}^{*}(\mathbf{s}) Q=0,
$$

for all $\mathbf{s} \in \mathcal{L}$. An example of such a map is the Killing form of a group. Such a map will be called a bi-invariant form in the following. Given such a bi-invariant form, it is simple to see that it will satisfy the following,

$$
\{\mathbf{s}, Q \mathbf{z}\}=Q[\mathbf{s}, \mathbf{z}],
$$

for arbitrary $\mathbf{s}, \mathbf{z} \in \mathcal{L}$.

Lemma II.1. If a Lie algebra is the direct sum of two subspaces $\mathcal{L}=\mathcal{L}_{0} \oplus \mathcal{L}_{1}$ and $Q$ is a non-singular bi-invariant form on $\mathcal{L}$ then $Q$ induces a splitting on the dual of the Lie algebra, $\mathcal{L}^{*}=\mathcal{L}_{0}^{*} \oplus \mathcal{L}_{1}^{*}$.

Proof: The two subspaces are defined by,

$$
\mathcal{L}_{0}^{*}=\left\{\mathbf{p} \in \mathcal{L}^{*}: \mathbf{p}=Q \mathbf{s} \text { for some } \mathbf{s} \in \mathcal{L}_{0}\right\}
$$

and

$$
\mathcal{L}_{1}^{*}=\left\{\mathbf{p} \in \mathcal{L}^{*}: \mathbf{p}=Q \mathbf{s} \text { for some } \mathbf{s} \in \mathcal{L}_{1}\right\}
$$

Theorem II.2. Suppose the splitting of the Lie algebra is a Cartan algebra, that is it satisfies the relations (34)-(36) given below, then the following relations hold for the coadjoint representation acting on the dual of the Lie algebra,

$$
\begin{array}{lll}
\left\{\mathcal{L}_{0}, \mathcal{L}_{0}^{*}\right\} & \subset & \mathcal{L}_{0}^{*}, \\
\left\{\mathcal{L}_{0}, \mathcal{L}_{1}^{*}\right\} & \subset & \mathcal{L}_{1}^{*}, \\
\left\{\mathcal{L}_{1}, \mathcal{L}_{0}^{*}\right\} & \subset & \mathcal{L}_{1}^{*}, \\
\left\{\mathcal{L}_{1}, \mathcal{L}_{1}^{*}\right\} & \subset & \mathcal{L}_{0}^{*}
\end{array}
$$

Proof: Consider arbitrary elements $\mathbf{s}_{i} \in \mathcal{L}_{i}$ and $\mathbf{p}_{j} \in \mathcal{L}_{j}^{*}$ where $i, j=0$ or 1 , then

$$
\left\{\mathbf{s}_{i}, \mathbf{p}_{j}\right\}=\left\{\mathbf{s}_{i}, Q \mathbf{s}_{j}\right\}=Q\left[\mathbf{s}_{i}, \mathbf{s}_{j}\right]
$$

for some $\mathbf{s}_{j} \in \mathcal{L}_{j}$. Finally, since $\left[\mathbf{s}_{i}, \mathbf{s}_{j}\right] \in \mathcal{L}_{i+j(\bmod 2)}$, by equations (5) and (6) we have that $\left\{\mathbf{s}_{i}, \mathbf{p}_{j}\right\} \in \mathcal{L}_{i+j}^{*}(\bmod 2)$.

\section{GeOMetric Control AND BRocketT's TheOrem}

The geometric treatment of optimal control theory can be found in standard texts such as [8]. A very clear exposition of the use of the Pontryagin maximum principle on Lie groups can be found in [14], and it is this reference that will be followed most closely below. The state equation of a leftinvariant system is,

$$
\dot{G}(t)=G(t) U(t) .
$$

Here $G(t)$ is a curve in the Lie group and $U(t)$ is a curve in the group's Lie algebra representing a control action.

The next step, according to [14], is to write down the coadjoint equation of the system. Unfortunately, only the case of right-invariant systems is given. However, it is a simple matter to change. It is not too difficult to see that the coadjoint equation for a left-invariant system is,

$$
\dot{\mathbf{p}}(t)=\mathbf{p}(t) \circ \operatorname{ad}(\mathbf{u}(t)),
$$

where, as usual, $\mathbf{u}$ is the vector representing the Lie algebra matrix $U$ and $\circ$ denotes composition of maps. Notice that the only difference between this and the original relation for a right-invariant systems given in [14], is the disappearance of a minus sign.

Lemma III.1. The coadjoint equation can be written,

$$
\dot{\mathbf{p}}(t)=-\{\mathbf{u}(t), \mathbf{p}(t)\} .
$$

Proof: For an arbitrary vector $\mathbf{s}$ in the Lie algebra we have,

$$
\begin{aligned}
& \dot{\mathbf{p}}(t)^{T} \mathbf{s}=\mathbf{p}(t) \circ \operatorname{ad}(\mathbf{u}(t)) \mathbf{s}=\mathbf{p}(t)^{T}[\mathbf{u}(t), \mathbf{s}] \\
&=-\{\mathbf{u}(t), \mathbf{p}(t)\}^{T} \mathbf{s}
\end{aligned}
$$

using equation (2) above. Since $\mathbf{s}$ is arbitrary it may be cancelled.

Next we introduce the objective function,

$$
\Phi=\frac{1}{2} \int_{0}^{1} \mathbf{u}^{T} Q \mathbf{u} \mathrm{d} t,
$$

where $Q$ is the matrix of a bi-invariant form, as introduced above. The Hamiltonian for the system is thus,

$$
H=\mathbf{p}^{T} \mathbf{u}+\frac{1}{2} \mathbf{u}^{T} Q \mathbf{u} .
$$

Along an optimal trajectory the Hamiltonian is constant so that,

$$
\mathbf{p}=-Q \mathbf{u} .
$$

Remark III.1. Care must be taken here. The bi-invariant form $Q$ is not necessarily positive or negative definite. Hence, the solutions found here might not minimise or maximise the objective function. All that can be said is that the solutions are stationary for the system. On a compact semi-simple Lie group the Killing form is known to be negative definite. So if the group under consideration was compact and semi-simple and $Q$ was the Killing form then it could be concluded that the solution maximises $\Phi$. However, the examples treated below are systems on the group of rigid-body displacements $S E(3)$ and this group is neither compact nor semi-simple and is know not to have any bi-invariant forms which are positive or negative definite.

The specification of the system to be considered is completed by assuming that $\mathbf{u}(t) \in \mathcal{L}_{1}$ for all $t$. That is the control action is a vector in a Lie triple system.

Theorem III.2. (After Brockett 1999) Consider a system as defined above; that is a left-invariant system defined by (11) with an objective function as given in (15) and subject to the restriction that the controls $\mathbf{u}(t)$ lie in a Lie triple system. The stationary solution to such a system is given by,

$$
G(t)=G(0) e^{t\left(Z_{1}-Z_{0}\right)} e^{t Z_{0}} .
$$

where $\mathbf{z}_{1} \in \mathcal{L}_{1}$ and $\mathbf{z}_{0} \in \mathcal{L}_{0}$ are constants and $G(0)$ is the initial value of the system, hence an element of the 
group. Further, the control law which produces this stationary trajectory is given by,

$$
U(t)=e^{-t Z_{0}} Z_{1} e^{t Z_{0}}
$$

Proof: Let $\mathbf{p}(t)=\mathbf{p}_{0}(t)+\mathbf{p}_{1}(t)$ where $\mathbf{p}_{0}(t) \in \mathcal{L}_{0}^{*}$ and $\mathbf{p}_{1}(t) \in \mathcal{L}_{1}^{*}$. Using the results of theorem II.2, the coadjoint equations become,

$$
\begin{aligned}
& \dot{\mathbf{p}}_{0}(t)=-\left\{\mathbf{u}(t), \mathbf{p}_{1}(t)\right\}, \\
& \dot{\mathbf{p}}_{1}(t)=-\left\{\mathbf{u}(t), \mathbf{p}_{0}(t)\right\} .
\end{aligned}
$$

From the Hamiltonian, equation (17), we have,

$$
\mathbf{p}_{1}(t)=-Q \mathbf{u}(t) \text {. }
$$

Substituting this into equation (20) gives

$$
\dot{\mathbf{p}}_{0}(t)=\{\mathbf{u}(t), Q \mathbf{u}(t)\}=Q[\mathbf{u}(t), \mathbf{u}(t)]=0 .
$$

Hence $\mathbf{p}_{0}(t)=\mathbf{k}_{0}$ a constant. Substituting into equation (21) gives,

$$
\dot{\mathbf{p}}_{1}(t)=-Q \dot{\mathbf{u}}(t)=-\left\{\mathbf{u}(t), \mathbf{k}_{0}\right\} .
$$

As $Q$ is non-singular we can write the above equation as,

$$
\dot{\mathbf{u}}(t)=\left[\mathbf{u}(t), Q^{-1} \mathbf{k}_{0}\right] .
$$

This equation is familiar from the theory of Lax pairs and hence is easily solved. Writing $\mathbf{z}_{0}=Q^{-1} \mathbf{k}_{0}$ for the constant twist, the solution is,

$$
\mathbf{u}(t)=e^{-\operatorname{ad}\left(\mathbf{z}_{0}\right) t} \mathbf{z}_{1} .
$$

In a matrix representation this can be written as,

$$
U(t)=e^{-t Z_{0}} Z_{1} e^{t Z_{0}}
$$

where $Z_{1}$ is another constant Lie algebra element found from the initial conditions. Notice that we must have $\mathbf{z}_{1} \in \mathcal{L}_{1}$, since by hypothesis the controls are restricted to the Lie triple system $\mathbf{u}(t) \in \mathcal{L}_{1}$, this must hold when $t=0$ in particular. This gives the stationary control. The original kinematic equation for the vehicle can now be written,

$$
\dot{G}(t)=G(t) e^{-t Z_{0}} Z_{1} e^{t Z_{0}}
$$

The solution to this is simply,

$$
G(t)=G(0) e^{t\left(Z_{1}-Z_{0}\right)} e^{t Z_{0}} .
$$

This is easily verified by substitution. For a derivation, the original argument given in [3] can be easily modified.

Remark III.2. The stationary solutions are independent of $Q$ in the sense that if there is a choice of different bi-invariant forms $Q$, the solutions they give will be the same. In some cases there will be only one such bi-invariant form $Q$, see for example section V-A below. In some cases however there may be a choice of different bi-invariant forms. A similar situation happens with geodesics of bi-invariant (pseudo)-metrics on Lie groups. If the group has several bi-invariant metrics, then the geodesics for all these metrics are the same, the only difference is that the 'distance' along a geodesic will be different for the different metrics.

Remark III.3. In kinematics there is a long tradition of naming special rigid-body motions after people. Hence, in consideration of the discussion in the introduction above, it is proposed that this be called the Brockett motion.

\section{The Lie Triple Systems of $S E(3)$}

The considerations above have been of a general nature and apply to any Lie group. Following a review of triple systems in general, the rest of this work the focus is on the group of rigidbody displacements $S E(3)$. In this section a classification of the Lie triple systems of $S E(3)$ is derived.

\section{A. Definitions}

There are at least three ways to define a Lie triple system and unfortunately they give slightly different results. The first definition is perhaps the simplest.

Definition IV.1. Let $\mathcal{L}$ be a Lie algebra and $\mathcal{L}_{1}$ a linear subspace of $\mathcal{L}$. The space $\mathcal{L}_{1}$ is a Lie triple system if and only if it is closed under double brackets. That is, if $X, Y, Z \in \mathcal{L}_{1}$ then $[X,[Y, Z]] \in \mathcal{L}_{1}$.

Unfortunately, under this definition, subalgebras would be considered Lie triple systems since they are closed under the bracket operation they are automatically closed under double brackets.

Using this definition however, there are two subalgebras associated to every Lie Triple system.

Lemma IV.1. Given a Lie triple $\mathcal{L}_{1}$ the linear space spanned by all brackets of elements of $\mathcal{L}_{1}$ is a Lie algebra. This algebra is often denoted $\left[\mathcal{L}_{1}, \mathcal{L}_{1}\right]$ and called the derived subalgebra.

Proof: To see that the set of the bracket elements of $\mathcal{L}_{1}$ is closed under the bracket we can use the Jacobi identity,

$$
\begin{aligned}
{[[X, Y],[Z, W]] } & =-[Z,[W,[X, Y]]]-[W[[X, Y], Z]] \\
& =-[Z, U]-[W, V],
\end{aligned}
$$

where $U=[W,[X, Y]]$ and $V=[[X, Y], Z]$ and by the definition of the Lie triple system above these are elements of $\mathcal{L}_{1}$.

Lemma IV.2. The linear space spanned by $\mathcal{L}_{1}$ and the derived subalgebra is also a Lie algebra, denoted $\mathcal{L}_{1}+\left[\mathcal{L}_{1}, \mathcal{L}_{1}\right]$.

Proof: To see that this is a subalgebra notice that we have already seen that $\left[\mathcal{L}_{1}, \mathcal{L}_{1}\right]$ is closed with respect to the bracket operation and also by definition the bracket of any pair of elements from $\mathcal{L}_{1}$ is an element of $\left[\mathcal{L}_{1}, \mathcal{L}_{1}\right]$. The only case that remains to check is where we take the bracket of a element from $\mathcal{L}_{1}$ with one from $\left[\mathcal{L}_{1}, \mathcal{L}_{1}\right]$. A typical element from $\left[\mathcal{L}_{1}, \mathcal{L}_{1}\right]$ is a linear sum of elements of the form $[Y, Z]$ with $Y, Z \in \mathcal{L}_{1}$. Hence if $X \in \mathcal{L}_{1}$ we have, $[X,[Y, Z]] \in \mathcal{L}_{1}$ from the definition of the Lie triple system.

Let us relabel the derived subalgebra as $\mathcal{L}_{0}=\left[\mathcal{L}_{1}, \mathcal{L}_{1}\right]$. This leads to the following relations for elements of $\mathcal{L}_{0}$ and $\mathcal{L}_{1}$,

Theorem IV.3. Let $A, B \in \mathcal{L}_{0}$ and $X, Y \in \mathcal{L}_{1}$, then

$$
\begin{aligned}
& {[A, B] \in \mathcal{L}_{0},} \\
& {[A, X] \in \mathcal{L}_{1},} \\
& {[X, Y] \in \mathcal{L}_{0} .}
\end{aligned}
$$


These relations are often expressed by the following notation

$$
\begin{aligned}
& {\left[\mathcal{L}_{0}, \mathcal{L}_{0}\right] \subset \mathcal{L}_{0},} \\
& {\left[\mathcal{L}_{0}, \mathcal{L}_{1}\right] \subset \mathcal{L}_{1},} \\
& {\left[\mathcal{L}_{1}, \mathcal{L}_{1}\right] \subset \mathcal{L}_{0} .}
\end{aligned}
$$

Proof: The first relation here follows from the closure of the derived subalgebra, Lemma IV.1. The second is the definition of the Lie triple system. The third is the definition of the derived subalgebra.

This leads to our second definition:

Definition IV.2. Let $\mathcal{L}$ be a Lie algebra and $\mathcal{L}_{0}$ and $\mathcal{L}_{1}$ be a pair of linear subspaces of $\mathcal{L}$ satisfying the relations (34)-(36), above, then $\mathcal{L}_{1}$ is called a Lie Triple System.

Remark IV.1. The two definitions above are not quite equivalent. Certainly by theorem IV.3 any Lie triple system satisfying definition IV.1 also satisfies definition IV.2. Conversely however, a Lie triple system which satisfies definition IV.2 may not satisfies definition IV.1 in the sense that we can only be sure that $\left[\mathcal{L}_{1}, \mathcal{L}_{1}\right] \subset \mathcal{L}_{0}$, not that $\mathcal{L}_{0}=\left[\mathcal{L}_{1}, \mathcal{L}_{1}\right]$. The subspace $\mathcal{L}_{0}$ is a subalgebra by condition (34), but there may be several different possibilities satisfying the conditions as will be seen below. From now on $\mathcal{L}_{0}$ will be referred to as a complementary subalgebra. However, it is clear that a subspace $\mathcal{L}_{1}$ which is a Lie triple system by definition IV.2 must also be a Lie triple system by definition IV.1.

To exclude the cases where $\mathcal{L}_{1}$ is itself a subalgebra we could require that $\mathcal{L}_{0}$ and $\mathcal{L}_{1}$ satisfy $\mathcal{L}_{0} \cap \mathcal{L}_{1}=\{\mathbf{0}\}$. That is we change the definition above by just one word: “... $\mathcal{L}_{0}$ and $\mathcal{L}_{1}$ be a pair of disjoint linear subspaces of $\mathcal{L} \ldots$.." Now the subalgebra $\mathcal{L}_{1}+\left[\mathcal{L}_{1}, \mathcal{L}_{1}\right]$ is the direct sum, $\mathcal{L}_{0} \oplus \mathcal{L}_{1}$. However, this does not exclude the possibility that $\mathcal{L}_{1}$ is a commutative subalgebra of $\mathcal{L}$, see below.

With this definition, the pair $\mathcal{L}_{0}, \mathcal{L}_{1}$ is sometimes called a Cartan pair of the algebra. In [3] these relations are used to define the subalgebra $\mathcal{L}_{0} \oplus \mathcal{L}_{1}$, as a $\mathbb{Z}_{2}$-graded subalgebra. Notice that this subalgebra may or may not be all of $\mathcal{L}$. All we can say is that $\mathcal{L}_{0} \oplus \mathcal{L}_{1} \subseteq \mathcal{L}$. It will be this definition of a Lie triple system that is used in the following.

The last definition we look at here is only included for completeness. It shows that the idea of a Lie triple system can be defined without reference to any Lie algebraic superstructure.

Definition IV.3. A Lie triple system $\mathcal{L}_{1}$, is a vector space together with a tri-linear ternary operator $[X, Y, Z]$ which satisfies the following relations,

$$
\begin{gathered}
{[X, Y, Y]=\mathbf{0}} \\
{[X, Y, Z]+[Y, Z, X]+[Z, X, Y]=\mathbf{0}} \\
{[A, B,[X, Y, Z]]=[[A, B, X], Y, Z]+[X,[A, B, Y], Z]} \\
+[X, Y,[A, B, Z]]
\end{gathered}
$$

For any $A, B, X, Y, Z \in \mathcal{L}_{1}$.

It is easy to see that if $\mathcal{L}_{1}$ is a Lie triple system as defined by the previous definitions as a subspace of a Lie algebra with ternary operation given by the double bracket $[X, Y, Z]=$

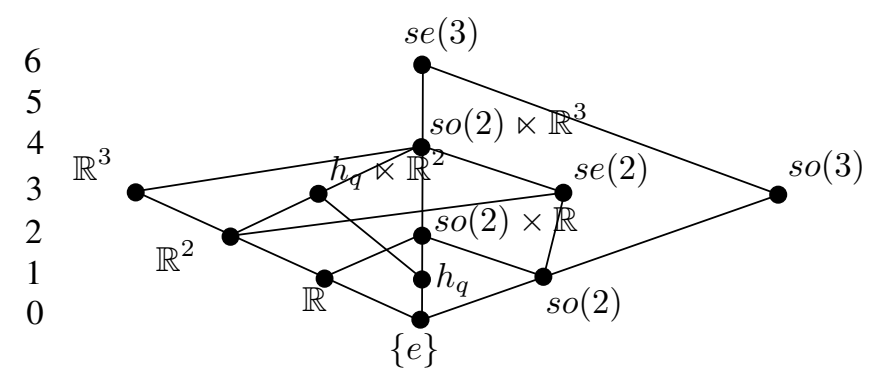

Fig. 1. Hasse diagram for the Lattice of subalgebras of $s e(3)$. The numbers on the left give the dimensions of the subalgebras at that level.

$[X,[Y, Z]]$ then the above relations will be satisfied. The first relation here is the anti-symmetry of the bracket.

However, it is also possible to show that a Lie triple system given by the above definition can always be embedded in a Lie algebra hence this elegant definition doesn't give us anything we can't get from the previous definitions. See [7].

Finally here, consider a commutative subalgebra of $\mathcal{L}$. The derived algebra of such a subspace will be the zero vector $\{\mathbf{0}\}$, by definition. The other conditions for a Lie Triple system will be satisfied however. This is a rather degenerate situation, where $\mathcal{L}_{0}=\{\boldsymbol{0}\}$ and $\mathcal{L}_{1}$ is commutative. This situation will usually be ignored as a trivial case, however, definition IV.2 above only requires that the derived subalgebra is a subset of $\mathcal{L}_{0}$ hence it allows for the case where $\mathcal{L}_{1}$ is a commutative subalgebra but $\mathcal{L}_{0}$ is a non-trivial subalgebra which acts linearly on $\mathcal{L}_{1}$.

\section{B. Classification}

Fortunately for us there is a classification of all linear subspaces of the Lie algebra se(3) up to rigid displacements. These linear subspaces are known as screw systems in the robotics and mechanisms literature, see the appendix and references therein for further details.

Hence to find the Lie triple systems all we need to do is to inspect the list of screw systems and check which are Lie triple systems. Actually, since we know which of these systems are subalgebras we can make the search a little shorter by taking each subalgebra in turn and examining its complimentary subspaces. There may be more than one complimentary subspace in the sense that the $\mathcal{L}_{0} \oplus \mathcal{L}_{1}$ subalgebra could be any subalgebra containing the one we are looking at, not just $s e(3)$ itself. The Hasse diagram for the lattice of subalgebras of $s e(3)$ is given in Fig. 1. From this it is straightforward to draw up a table of possible complimentary subalgebras and their possible $\mathcal{L}_{0} \oplus \mathcal{L}_{1}$ subalgebras, that is the possible superalgebras containing the complementary subalgebra, see table I.

This gives 31 possible cases to check. By inspection, eighteen of these cases satisfy definition IV.2, see table II. In some of these cases however, the action of $\mathcal{L}_{0}$ on $\mathcal{L}_{1}$ is trivial, that is $\left[\mathcal{L}_{0}, \mathcal{L}_{1}\right]=\mathbf{0}$. These cases are are marked with an asterisk in the $\operatorname{dim} \mathcal{L}_{1}$ column.

The case where $\mathbb{R}$ is a subalgebra of $s o(2) \ltimes \mathbb{R}^{3}$ will be used to demonstrate the techniques used to draw up this table. We can take $\mathcal{L}_{0}=\mathbb{R}$ as generated by the single pure translational 
TABLE I

THE POSSIBLE SUBALGEBRAS AND THEIR SUPERALGEBRAS IN $s e(3)$. (HERE $\ltimes$ DENOTES THE SEMI-DIRECT PRODUCT AND $h_{q}$ IS THE GROUP OF SCREW DISPLACEMENTS ABOUT A FIXED AXIS WITH FIXED PITCH $q$.)

\begin{tabular}{|c|c|}
\hline Subalgebra $\mathcal{L}_{0}$ & Possible $\mathcal{L}_{0} \oplus \mathcal{L}_{1}$ subalgebras \\
\hline$s o(2) \ltimes \mathbb{R}^{3}$ & $s e(3)$ \\
\hline so(3) & $s e(3)$ \\
\hline$s e(2)$ & $s o(2) \ltimes \mathbb{R}^{3}, \operatorname{se}(3)$ \\
\hline$h_{q} \ltimes \mathbb{R}^{2}$ & $s o(2) \ltimes \mathbb{R}^{3}, \operatorname{se}(3)$ \\
\hline $\mathbb{R}^{3}$ & $s o(2) \ltimes \mathbb{R}^{3}, \operatorname{se}(3)$ \\
\hline$s o(2) \times \mathbb{R}$ & $s o(2) \ltimes \mathbb{R}^{3}, \operatorname{se}(3)$ \\
\hline $\mathbb{R}^{2}$ & $\mathbb{R}^{3}, h_{q} \ltimes \mathbb{R}^{2}, \operatorname{se}(2)$, so $(2) \ltimes \mathbb{R}^{3}, \operatorname{se}(3)$ \\
\hline$s o(2)$ & $s o(3)$, so $(2) \times \mathbb{R}, s e(2)$, so $(2) \times \mathbb{R}^{3}$, se $(3)$ \\
\hline$h_{q}$ & $h_{q} \ltimes \mathbb{R}^{2}, s o(2) \times \mathbb{R}, s o(2) \ltimes \mathbb{R}^{3}, s e(3)$ \\
\hline $\mathbb{R}$ & $\begin{array}{l}\text { so }(2) \times \mathbb{R}, \mathbb{R}^{2}, \text { se }(2), h_{q} \ltimes \mathbb{R}^{2}, \mathbb{R}^{3}, \\
\text { so }(2) \times \mathbb{R}^{3}, \text { se }(3)\end{array}$ \\
\hline
\end{tabular}

twist $\left(\begin{array}{l}\mathbf{0} \\ \mathbf{i}\end{array}\right)$. Next we can assume that $\mathcal{L}_{1}$ is generated be the twists, $\left\{\left(\begin{array}{c}\boldsymbol{\omega} \\ \mathbf{0}\end{array}\right),\left(\begin{array}{c}\mathbf{0} \\ \mathbf{v}_{1}\end{array}\right),\left(\begin{array}{c}\mathbf{0} \\ \mathbf{v}_{2}\end{array}\right)\right\}$. Here $\boldsymbol{\omega}$ is fixed, but to be determined. Similarly, $\mathbf{v}_{1}$ and $\mathbf{v}_{2}$ are to be determined but must be linearly independent and linearly independent from $\mathbf{i}$. From equation (35) we have that,

$$
\mathbf{i} \times \boldsymbol{\omega}=\lambda \mathbf{v}_{1}+\mu \mathbf{v}_{2}
$$

for some constants $\lambda, \mu$. This tells us that the vectors $\mathbf{v}_{1}, \mathbf{v}_{2}$, can have no component in the $\mathbf{i}$ direction; $\mathbf{i} \cdot \mathbf{v}_{1}=\mathbf{i} \cdot \mathbf{v}_{2}=0$. So as a basis for $\mathcal{L}_{1}$ we can take, $\left\{\left(\begin{array}{l}\boldsymbol{\omega} \\ \mathbf{0}\end{array}\right),\left(\begin{array}{l}\mathbf{0} \\ \mathbf{j}\end{array}\right),\left(\begin{array}{l}\mathbf{0} \\ \mathbf{k}\end{array}\right)\right\}$.

Next consider equation (36),

$$
\left[\left(\begin{array}{l}
\boldsymbol{\omega} \\
\mathbf{0}
\end{array}\right),\left(\begin{array}{l}
\mathbf{0} \\
\mathbf{j}
\end{array}\right)\right]=\left(\begin{array}{c}
\mathbf{0} \\
\boldsymbol{\omega} \times \mathbf{j}
\end{array}\right) .
$$

For this to be in $\mathcal{L}_{0}$ we must have $\boldsymbol{\omega}=\alpha \mathbf{j}+\beta \mathbf{k}$ for some constants $\alpha$ and $\beta$ not both zero. Notice that the solution $\boldsymbol{\omega}=$ $\mathbf{0}$ is not possible since in that case $\mathcal{L}_{0} \oplus \mathcal{L}_{1} \neq s o(2) \ltimes \mathbb{R}^{3}$. Finally it is simple to check that,

$$
\left[\left(\begin{array}{l}
\boldsymbol{\omega} \\
\mathbf{0}
\end{array}\right),\left(\begin{array}{l}
\mathbf{0} \\
\mathbf{k}
\end{array}\right)\right] \in \mathcal{L}_{0}
$$

This confirms that this is a Lie triple system, or more precisely a Cartan pair of subalgebras. Note that by rotating around the $x$-axis this system can be transformed to the canonical form,

$$
\mathcal{L}_{0}=\operatorname{Span}\left\{\left(\begin{array}{l}
\mathbf{0} \\
\mathbf{i}
\end{array}\right)\right\}, \quad \mathcal{L}_{1}=\operatorname{Span}\left\{\left(\begin{array}{l}
\mathbf{k} \\
\mathbf{0}
\end{array}\right),\left(\begin{array}{l}
\mathbf{0} \\
\mathbf{j}
\end{array}\right),\left(\begin{array}{l}
\mathbf{0} \\
\mathbf{k}
\end{array}\right)\right\} .
$$

Table II contains many duplicates for possible Lie triple systems, $\mathcal{L}_{1}$. The explanation is that if some $\mathcal{L}_{1}$ is a Lie triple system with a complementary subalgebra $\mathcal{L}_{0}$, then it may also be a Lie triple system with some subalgebra of $\mathcal{L}_{0}$ as complementary subalgebra. Certainly, if $\mathcal{L}_{1}$ carries an action of $\mathcal{L}_{0}$ then it carries an action of a subalgebra of $\mathcal{L}_{0}$. In fact the table really lists the possible Cartan pairs in $s e(3)$. It would perhaps be better to present a list of Lie triple systems giving the maximal subalgebras $\mathcal{L}_{0}$. This is done in table III.
TABLE II

THE CARTAN PAIRS OF $s e(3)$. (AN ASTERISK IN THE THIRD COLUMN DENOTES THAT THE ACTION OF $\mathcal{L}_{0}$ ON $\mathcal{L}_{1}$ IS TRIVIAL. THE FOURTH COLUMN GIVES THE GIBSON-HUNT TYPE OF THE SCREW SYSTEM $\mathcal{L}_{1}$, SEE THE APPENDIX FOR FURTHER DETAILS.)

\begin{tabular}{|c|l|l|l|}
\hline $\mathcal{L}_{0}$ & $\mathcal{L}_{0} \oplus \mathcal{L}_{1}$ & $\operatorname{dim} \mathcal{L}_{1}$ & GH type of $\mathcal{L}_{1}$ \\
\hline \hline$s o(3)$ & $s e(3)$ & 3 & IID \\
\hline$s e(2)$ & $s o(2) \ltimes \mathbb{R}^{3}$ & $1^{*}$ & $p=\infty$ \\
\hline$s e(2)$ & $s e(3)$ & 3 & IIB $(p=0)$ \\
\hline$s o(2) \times \mathbb{R}$ & $s o(2) \ltimes \mathbb{R}^{3}$ & 2 & IIC \\
\hline$s o(2) \times \mathbb{R}$ & $s e(3)$ & 4 & $\overline{\text { IB }}{ }^{0}$ \\
\hline $\mathbb{R}^{2}$ & $\mathbb{R}^{3}$ & $1^{*}$ & $p=\infty$ \\
\hline$s o(2)$ & $s o(3)$ & 2 & IIA $(p=0)$ \\
\hline$s o(2)$ & $s o(2) \times \mathbb{R}$ & $1^{*}$ & $p=\infty$ \\
\hline$s o(2)$ & $s e(2)$ & 2 & IIC \\
\hline$s o(2)$ & $s o(2) \ltimes \mathbb{R}^{3}$ & 3 & IID \\
\hline$h_{q}$ & $h_{q} \ltimes \mathbb{R}^{2}$ & 2 & IIC \\
\hline$h_{q}$ & $s o(2) \times \mathbb{R}$ & $1^{*}$ & $p=-q$ \\
\hline $\mathbb{R}$ & $s o(2) \times \mathbb{R}$ & $1^{*}$ & $p=0$ \\
\hline $\mathbb{R}$ & $\mathbb{R}^{2}$ & $1^{*}$ & $p=\infty$ \\
\hline $\mathbb{R}$ & $s e(2)$ & 2 & IIB $(p=0)$ \\
\hline $\mathbb{R}$ & $h_{q} \ltimes \mathbb{R}^{2}$ & 2 & IIB $(p=q)$ \\
\hline $\mathbb{R}$ & $\mathbb{R}^{3}$ & $2^{*}$ & IIC \\
\hline $\mathbb{R}$ & $s o(2) \ltimes \mathbb{R}^{3}$ & 3 & IC ${ }^{*}$ \\
\hline
\end{tabular}

TABLE III

THE LiE TRIPLE Systems of se(3)

\begin{tabular}{|c|l|l|l|}
\hline $\operatorname{dim} \mathcal{L}_{1}$ & GH type of $\mathcal{L}_{1}$ & $\operatorname{maximal} \mathcal{L}_{0}$ & $\mathcal{L}_{0} \oplus \mathcal{L}_{1}$ \\
\hline \hline 4 & $\overline{\mathrm{IB}}{ }^{0}$ & $s o(2) \times \mathbb{R}$ & $s e(3)$ \\
\hline 3 & $\mathrm{IID}$ & $s o(3)$ & $s e(3)$ \\
\hline 3 & $\mathrm{IIB}(p=0)$ & $s e(2)$ & $s e(3)$ \\
\hline 3 & $\mathrm{IC} 0$ & $\mathbb{R}$ & $s o(2) \ltimes \mathbb{R}^{3}$ \\
\hline 2 & $\mathrm{IIC}$ & $s o(2) \times \mathbb{R}$ & $s o(2) \ltimes \mathbb{R}^{3}$ \\
\hline 2 & $\mathrm{IIA}(p=0)$ & $s o(2)$ & $s o(3)$ \\
\hline 2 & $\mathrm{IIB}(p=0)$ & $\mathbb{R}$ & $s e(2)$ \\
\hline 2 & $\mathrm{IIB}(p=q)$ & $\mathbb{R}$ & $h_{q} \ltimes \mathbb{R}^{2}$ \\
\hline $1^{*}$ & $p=\infty$ & $s e(2)$ & $s o(2) \ltimes \mathbb{R}^{3}$ \\
\hline $1^{*}$ & $p=-q$ & $h_{q}$ & $s o(2) \times \mathbb{R}$ \\
\hline $1^{*}$ & $p=0$ & $\mathbb{R}$ & $s o(2) \times \mathbb{R}$ \\
\hline
\end{tabular}

\section{EXAMPLES}

For controllability the completion of the triple system must be all of $s e(3),[4$, chap. 7]. The completion subalgebra is slightly different from the subalgebra $\mathcal{L}_{0} \oplus \mathcal{L}_{1}$ given in table III above, this is because $\mathcal{L}_{0}$ in the table is the maximal subalgebra which carries an action on $\mathcal{L}_{1}$. The completion subalgebra is given by $\mathcal{L}_{1}+\left[\mathcal{L}_{1}, \mathcal{L}_{1}\right]$. In the second row of table III, $\mathcal{L}_{1}$ is a commutative subalgebra and hence the bracket $\left[\mathcal{L}_{1}, \mathcal{L}_{1}\right]$ is the zero vector. In other words the completion 
subalgebra here is just $\mathcal{L}_{1}=\mathbb{R}^{3}$. So there are just two controllable Lie triple systems in $s e(3)$.

\section{A. Cars}

The first example considered here is a planar example, that is the Lie algebra under consideration is $\mathcal{L}=s e(2)$. However, this can be considered as a subalgebra of $s e(3)$ and the Lie triple system is given by the seventh row in table III. It is not necessary to use the full 6-dimensional formalism of $s e(3)$ however. The two subspaces of the Lie algebra can be written as,

$$
\mathcal{L}_{0}=\operatorname{Span}\left\{\left(\begin{array}{lll}
0 & 0 & 0 \\
0 & 0 & 1 \\
0 & 0 & 0
\end{array}\right)\right\}
$$

and

$$
\mathcal{L}_{1}=\operatorname{Span}\left\{\left(\begin{array}{ccc}
0 & -1 & 0 \\
1 & 0 & 0 \\
0 & 0 & 0
\end{array}\right),\left(\begin{array}{lll}
0 & 0 & 1 \\
0 & 0 & 0 \\
0 & 0 & 0
\end{array}\right)\right\} .
$$

The subspace $\mathcal{L}_{0}$ consists of translations in the $y$-direction and the Lie triple system is composed of rotations about the origin and translations in the $x$-direction.

$$
\begin{aligned}
& \text { Now suppose } Z_{0}=\lambda\left(\begin{array}{lll}
0 & 0 & 0 \\
0 & 0 & 1 \\
0 & 0 & 0
\end{array}\right) \text { and } \\
& Z_{1}=\omega\left(\begin{array}{ccc}
0 & -1 & 0 \\
1 & 0 & 0 \\
0 & 0 & 0
\end{array}\right)+\mu\left(\begin{array}{lll}
0 & 0 & 1 \\
0 & 0 & 0 \\
0 & 0 & 0
\end{array}\right) .
\end{aligned}
$$

The exponentials of these matrices can be found using the results in $[10, \S 5.3]$,

$$
e^{Z_{0} t}=I_{3}+\lambda t\left(\begin{array}{ccc}
0 & 0 & 0 \\
0 & 0 & 1 \\
0 & 0 & 0
\end{array}\right)=\left(\begin{array}{ccc}
1 & 0 & 0 \\
0 & 1 & \lambda t \\
0 & 0 & 1
\end{array}\right) .
$$

So the stationary control is given by,

$$
U(t)=e^{-Z_{0} t} Z_{1} e^{Z_{0} t}=\left(\begin{array}{ccc}
0 & -\omega & \mu-\lambda \omega t \\
\omega & 0 & 0 \\
0 & 0 & 0
\end{array}\right) .
$$

Notice that this control varies linearly with $t$.

The exponential of the first factor in the solution is given by,

$$
\begin{aligned}
e^{\left(Z_{1}-Z_{0}\right) t}=I_{3}+\sin (\omega t) & \left(\begin{array}{ccc}
0 & -1 & \frac{\mu}{\omega} \\
1 & 0 & \frac{-\lambda}{\omega} \\
0 & 0 & 0
\end{array}\right)+ \\
& (1-\cos (\omega t))\left(\begin{array}{ccc}
-1 & 0 & \frac{\lambda}{\omega} \\
0 & -1 & \frac{\mu}{\omega} \\
0 & 0 & 0
\end{array}\right) .
\end{aligned}
$$

That is,

$$
\begin{aligned}
& e^{\left(Z_{1}-Z_{0}\right) t}= \\
& \left(\begin{array}{ccc}
\cos (\omega t) & -\sin (\omega t) & \frac{\lambda}{\omega}-\frac{\lambda}{\omega} \cos (\omega t)+\frac{\mu}{\omega} \sin (\omega t) \\
\sin (\omega t) & \cos (\omega t) & \frac{\mu}{\omega}-\frac{\mu}{\omega} \cos (\omega t)-\frac{\lambda}{\omega} \sin (\omega t) \\
0 & 0 & 1
\end{array}\right) .
\end{aligned}
$$

Now assume that the motion begins at the identity so that $G(0)=I_{3}$. The explicit stationary motion will be given by,

$$
G(t)=e^{\left(Z_{1}-Z_{0}\right) t} e^{Z_{0} t}=\left(\begin{array}{ccc}
\cos (\omega t) & -\sin (\omega t) & \xi \\
\sin (\omega t) & \cos (\omega t) & \eta \\
0 & 0 & 1
\end{array}\right)
$$

where,

$$
\begin{aligned}
& \xi=\frac{\lambda}{\omega}-\frac{\lambda}{\omega} \cos (\omega t)-\lambda t \sin (\omega t)+\frac{\mu}{\omega} \sin (\omega t), \\
& \eta=\frac{\mu}{\omega}-\frac{\mu}{\omega} \cos (\omega t)-\frac{\lambda}{\omega} \sin (\omega t)+\lambda t \cos (\omega t) .
\end{aligned}
$$

Recall that this motion is the motion of the Frenet frame of a curve, [12]. The curve is the trajectory of the origin of the frame. It is possible to show that the curvature $\kappa$, of this curve is given by,

$$
\kappa=\frac{\omega}{\mu-\lambda \omega t} .
$$

When $\mu=0$ the curves are involutes of circles.

It may seem at first curious that the solution to this problem does not depend on which quadratic form is chosen in the objective function. The explanation in this case at least, is there is not really any choice. Suppose we write the Lie triple system in the 6-vector notation as,

$$
\mathcal{L}_{1}=\operatorname{Span}\left\{\left(\begin{array}{l}
\mathbf{k} \\
\mathbf{0}
\end{array}\right),\left(\begin{array}{l}
\mathbf{0} \\
\mathbf{i}
\end{array}\right)\right\} .
$$

Then, with $\omega$ and $\mu$ as arbitrary constants, the integrand of the performance integral on an arbitrary element of $\mathcal{L}_{1}$ will be,

$$
\mathbf{z}_{1}^{T} Q \mathbf{z}_{1}=\left(\begin{array}{c}
\omega \mathbf{k} \\
\mu \mathbf{i}
\end{array}\right)^{T}\left(\begin{array}{cc}
2 \beta I_{3} & \alpha I_{3} \\
\alpha I_{3} & 0
\end{array}\right)\left(\begin{array}{c}
\omega \mathbf{k} \\
\mu \mathbf{i}
\end{array}\right)=2 \beta \omega^{2} .
$$

That is, there is no dependence on $\alpha$. Since this objective function is positive semi-definite we can conclude that the motions found minimise this performance measure; the motions minimise the total angle turned by the car.

\section{B. Interpolation with Cars}

The solution above gives a three-parameter family of stationary motions depending on the parameters $\omega, \lambda$ and $\mu$. This means that it is a simple matter to use these motions to interpolate between different positions of the car.

At $t=0$ the position and orientation of the car is given by $G(0)=I_{3}$ that is, a standard or home configuration. From the above it should be possible to choose suitable values of the parameters to achieve a desired configuration when $t=1$ say. As an example consider how to achieve a translation to the point $(x, y)$ with a rotation of $\pi / 2$ radians. Clearly we must have $\omega=\pi / 2$. Solving for the other variables gives,

$$
\begin{aligned}
\lambda & =\left(\frac{\pi}{4-\pi}\right)(x-y), \\
\mu & =\left(\frac{\pi}{4-\pi}\right) x+\left(\frac{\pi(2-\pi)}{2(4-\pi)}\right) y .
\end{aligned}
$$

Some of these motions are illustrated in Fig. 2 for various values of $x$ and $y$. 


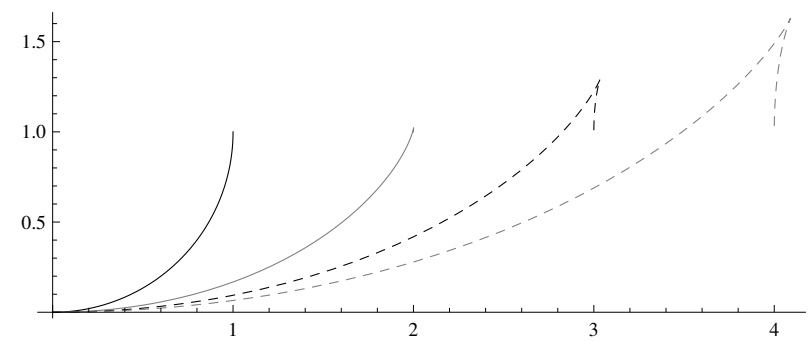

Fig. 2. Some Brockett motions of a car. The motions of a car can be given by a curve, the car is aligned with the tangent vector and normal to the curve with the origin of the moving frame following the curve. The black, grey, dashed black and dashed grey are designed to end at $(1,1),(2,1),(3,1)$ and $(4,1)$ respectively all with the car facing in the Positive $y$-direction.

In general, given the rotation angle, there will be a unique solution for motion from the home configuration to some other configuration. This is achieved by substituting for $\xi$ and $\eta$ when $t=1$ in (47). However, for some values of $\omega$ these equations are singular.

Suppose we wanted to achieve a unit translation in the $y$ direction. Clearly, to achieve the same orientation at $t=1$ as at $t=0$ we must have that $\omega=2 \pi$ or possibly some multiple of this. However, if it is required to minimize the rotation angle, as in equation (50) above then it is clear that $\omega= \pm 2 \pi$ should be chosen.

The equations for $\lambda$ and $\mu$ are then,

$$
\begin{aligned}
0 & =\frac{\lambda}{2 \pi}-\frac{\lambda}{2 \pi} \cos (2 \pi)-\lambda \sin (2 \pi)+\frac{\mu}{2 \pi} \sin (2 \pi), \\
1 & =\frac{\mu}{2 \pi}-\frac{\mu}{2 \pi} \cos (2 \pi)-\frac{\lambda}{2 \pi} \sin (2 \pi)+\lambda \cos (2 \pi) .
\end{aligned}
$$

The first equation above is an identity and the second is independent of $\mu$ but gives $\lambda=1$. Some example of these motions for cars are illustrated in Fig. 3.

However, there are final configurations which are not reachable with these motions. For example a pure translation of 1 unit in the $x$-direction and 1 unit in the $y$-direction cannot be achieved as the equations to be solved become singular and inconsistent. The solutions can also have cusps, indicating that the car must reverse its direction of travel.

\section{Bishop Motion}

A Bishop motion is a rigid-body motion where the body is fixed with respect to the Bishop frame of a smooth curve, [2]. These are very similar to the better known Frenet-Serret motions and are sometimes referred to as frame-rotation minimising motions or natural motions in the computer aided design literature. In [11] it was shown that Bishop motions can be characterised as left-invariant systems on $S E(3)$ where the control vector remains in a $\operatorname{IIB}(p=0)$ screw system. Above it was shown that this screw system is a Lie triple system; the third row in table III. Hence it should be possible to write down optimal Bishop motions.

In this case the subspaces of the Lie algebra are given by,

$$
\mathcal{L}_{0}=\operatorname{Span}\left\{\left(\begin{array}{l}
\mathbf{i} \\
\mathbf{0}
\end{array}\right),\left(\begin{array}{l}
\mathbf{0} \\
\mathbf{j}
\end{array}\right),\left(\begin{array}{l}
\mathbf{0} \\
\mathbf{k}
\end{array}\right)\right\}
$$

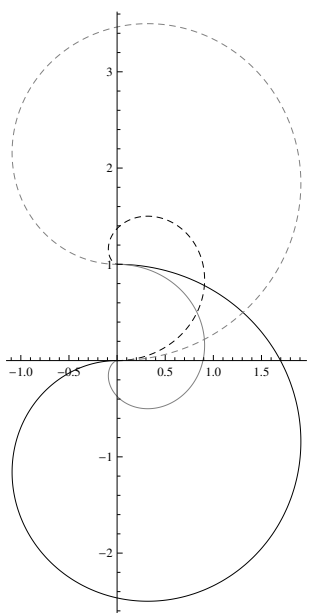

Fig. 3. Some Brockett Motions of a Car. The motions of a car can be given by a curve, the car is aligned with the tangent vector and normal to the curve with the origin of the moving frame following the curve. The parameter values for these motions are $\omega=2 \pi$ and $\lambda=1 / 2 \pi$ the different curves have different values of $\mu$, the black, grey, dashed black and dashed grey have the values $\mu=-1,0,1$ and 2 respectively.

and

$$
\mathcal{L}_{1}=\operatorname{Span}\left\{\left(\begin{array}{l}
\mathbf{0} \\
\mathbf{i}
\end{array}\right),\left(\begin{array}{l}
\mathbf{j} \\
\mathbf{0}
\end{array}\right),\left(\begin{array}{l}
\mathbf{k} \\
\mathbf{0}
\end{array}\right)\right\}
$$

The general Brockett motion here will have six arbitrary parameters. Hence it is difficult to write neatly and hence not very informative. To study these motions the link with the Bishop frame of a curve in space will be exploited.

It is simplest here to use the standard $4 \times 4$ representation of $S E(3)$, in this representation we can write,

$$
Z_{1}=v\left(\begin{array}{cccc}
0 & -k_{1} & -k_{2} & 1 \\
k_{1} & 0 & 0 & 0 \\
k_{2} & 0 & 0 & 0 \\
0 & 0 & 0 & 0
\end{array}\right)
$$

so that $v$ is the velocity of the curve and $k_{1}$ and $k_{2}$ are Bishop curvatures when $t=0$.

Notice that $\mathcal{L}_{0}$ is isomorphic to the Lie algebra $s e(2)$, hence the exponential $e^{Z_{0} t}$ where $Z_{0} \in \mathcal{L}_{0}$ is an arbitrary planar motion, which is either a uniform rotation about some point or a uniform translation.

In the case where $Z_{0}$ represents a uniform translation we can write,

$$
Z_{0}=\left(\begin{array}{cccc}
0 & 0 & 0 & 0 \\
0 & 0 & 0 & y \\
0 & 0 & 0 & z \\
0 & 0 & 0 & 0
\end{array}\right)
$$

The exponential of such a matrix is simply,

$$
e^{t Z_{0}}=\left(\begin{array}{cccc}
1 & 0 & 0 & 0 \\
0 & 1 & 0 & t y \\
0 & 0 & 1 & t z \\
0 & 0 & 0 & 0
\end{array}\right)
$$


Hence in this case stationary the control is,

$$
\begin{aligned}
U(t)=e^{-t Z_{0}} Z_{1} e^{t Z_{0}}= \\
v\left(\begin{array}{cccc}
0 & -k_{1} & -k_{2} & 1-\left(k_{1} y+k_{2} z\right) t \\
k_{1} & 0 & 0 & 0 \\
k_{2} & 0 & 0 & 0 \\
0 & 0 & 0 & 0
\end{array}\right) .
\end{aligned}
$$

So the curve determining the motion has a speed, $v(t)=v(1-$ $\left.\left(k_{1} y+k_{2} z\right) t\right)$ and Bishop curvatures, $k_{1}(t)=k_{1} /\left(1-\left(k_{1} y+\right.\right.$ $\left.\left.k_{2} z\right) t\right)$, and $k_{2}(t)=k_{2} /\left(1-\left(k_{1} y+k_{2} z\right) t\right)$. The curvature and torsion of the curve determining the motion are thus,

$$
\kappa=\frac{\sqrt{k_{1}^{2}+k_{2}^{2}}}{1-\left(k_{1} y+k_{2} z\right) t}, \text { and } \tau=\frac{d}{d t} \arctan \left(\frac{k_{2}}{k_{1}}\right)=0 .
$$

Hence these are plane curves. Notice also that these are also exactly the same plane curves as those found in section V-A above when,

$$
\frac{\mu}{\omega}=\frac{1}{\sqrt{k_{1}^{2}+k_{2}^{2}}} \quad \text { and } \quad \lambda=\frac{k_{1} y+k_{2} z}{\sqrt{k_{1}^{2}+k_{2}^{2}}} .
$$

In particular cases where explicit values of the parameters are given it is straightforward to determine the motion. This is facilitated by the explicit formula for the exponential,

$$
e^{S}=I_{4}+S+\frac{1}{\theta^{2}}(1-\cos \theta) S^{2}+\frac{1}{\theta^{3}}(\theta-\sin \theta) S^{3},
$$

where, $\theta^{2}=-(1 / 2) \operatorname{Tr}\left(S^{2}\right)$. See [10, chap. 4].

Next we turn to the case where the $s e(2)$ motion is a uniform rotation about a point. In this case $Z_{0}$ will be given by,

$$
Z_{0}=\omega\left(\begin{array}{cccc}
0 & 0 & 0 & 0 \\
0 & 0 & -1 & c_{z} \\
0 & 1 & 0 & -c_{y} \\
0 & 0 & 0 & 0
\end{array}\right),
$$

where $\left(c_{y}, c_{z}\right)$ is the coordinate of the rotation centre, and $\omega$ is the angular speed of the motion. The exponential of this is just,

$$
e^{Z_{0} t}=\left(\begin{array}{cccc}
1 & 0 & 0 & 0 \\
0 & \cos \omega t & -\sin \omega t & c_{y}-c_{y} \cos \omega t+c_{z} \sin \omega t \\
0 & \sin \omega t & \cos \omega t & c_{z}-c_{y} \sin \omega t-c_{z} \cos \omega t \\
0 & 0 & 0 & 1
\end{array}\right)
$$

The stationary control is then,

$$
U(t)=e^{-Z_{0} t} Z_{1} e^{Z_{0} t}=v\left(\begin{array}{cccc}
0 & -\omega_{3} & \omega_{2} & 1-\gamma t \\
\omega_{3} & 0 & 0 & 0 \\
-\omega_{2} & 0 & 0 & 0 \\
0 & 0 & 0 & 0
\end{array}\right),
$$

where,

$$
\begin{aligned}
\omega_{2} & =k_{1} \sin \omega t-k_{2} \cos \omega t, \\
\omega_{3}= & k_{1} \cos \omega t+k_{2} \sin \omega t, \\
\gamma & =\left(k_{1} c_{y}+k_{2} c_{z}\right)-\left(k_{1} c_{y}+k_{2} c_{z}\right) \cos \omega t+ \\
& \quad\left(k_{1} c_{z}-k_{2} c_{y}\right) \sin \omega t .
\end{aligned}
$$

An example of this type of motion is shown in Fig. 4.

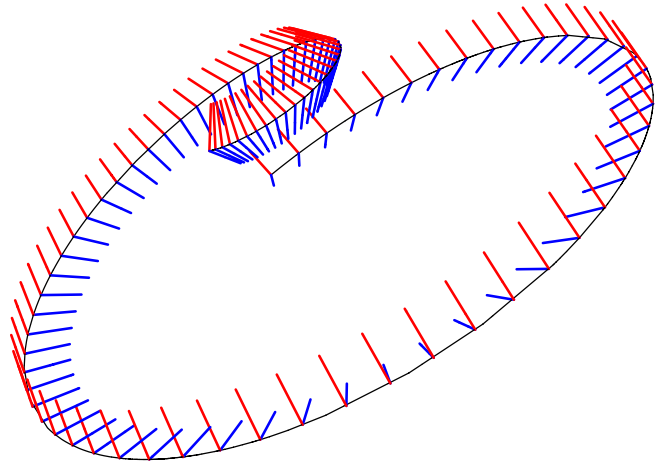

Fig. 4. A particular Brockett motion of Bishop type. The parameters for this motion are $\omega=1, v=2, k_{1}=3, k_{2}=4, c_{y}=1$ and $c_{z}=-1$. The red and blue lines represent the direction of the normals to the curve.

The curves which generate these motions have constant torsion, this can be seen as follows. Let, $\theta(t)=$ $\arctan \left(k_{2}(t) / k_{1}(t)\right)$, then by standard theory [2], the torsion of the curve is given by the derivative $\tau=d \theta(t) / d t$. For the curves defined above,

$$
\frac{k_{2}(t)}{k_{1}(t)}=\frac{k_{2} \cos \omega t-k_{1} \sin \omega t}{k_{1} \cos \omega t+k_{2} \sin \omega t} .
$$

Now, write $\sin \phi=k_{2} / \sqrt{k_{1}^{2}+k_{2}^{2}}$ and $\cos \phi=k_{1} / \sqrt{k_{1}^{2}+k_{2}^{2}}$, this allows us to see that,

$$
\theta(t)=\arctan \left(\frac{\sin (\phi-\omega t)}{\cos (\phi-\omega t)}\right)=\phi-\omega t+n \pi
$$

for some integer $n$. The derivative thus gives $\tau=-\omega$, a constant.

Finally here we look at the performance measure, with

$$
\mathbf{z}_{1}=\left(v\left(\begin{array}{l}
\mathbf{0} \\
\mathbf{i}
\end{array}\right)-v k_{2}\left(\begin{array}{l}
\mathbf{j} \\
\mathbf{0}
\end{array}\right)+v k_{1}\left(\begin{array}{l}
\mathbf{k} \\
\mathbf{0}
\end{array}\right)\right)
$$

this is given by,

$$
\mathbf{z}_{1}^{T} Q \mathbf{z}_{1}=2 \beta v^{2}\left(k_{1}^{2}+k_{2}^{2}\right) .
$$

Again we see that there is no dependence on $\alpha$, so there is really only one possible choice of objective function.

\section{D. $\overline{I B^{0}}$ Motion}

The motions presented in this section do not seem to be well know. They certainly fit into the general framework of vehicle motions as defined in [12] but do not appear to have been studied previously. This is possibly due to the fact that they do not correspond to the motions of any well-known vehicle. In this case the stationary solutions are rather lengthy to write down hence only the stationary controls will be recorded here.

The subspaces of the Lie algebra in this case are given by,

$$
\mathcal{L}_{0}=\operatorname{Span}\left\{\left(\begin{array}{l}
\mathbf{k} \\
\mathbf{0}
\end{array}\right),\left(\begin{array}{l}
\mathbf{0} \\
\mathbf{k}
\end{array}\right)\right\}
$$

and

$$
\mathcal{L}_{1}=\operatorname{Span}\left\{\left(\begin{array}{l}
\mathbf{i} \\
\mathbf{0}
\end{array}\right),\left(\begin{array}{l}
\mathbf{j} \\
\mathbf{0}
\end{array}\right),\left(\begin{array}{l}
\mathbf{0} \\
\mathbf{i}
\end{array}\right),\left(\begin{array}{l}
\mathbf{0} \\
\mathbf{j}
\end{array}\right)\right\}
$$




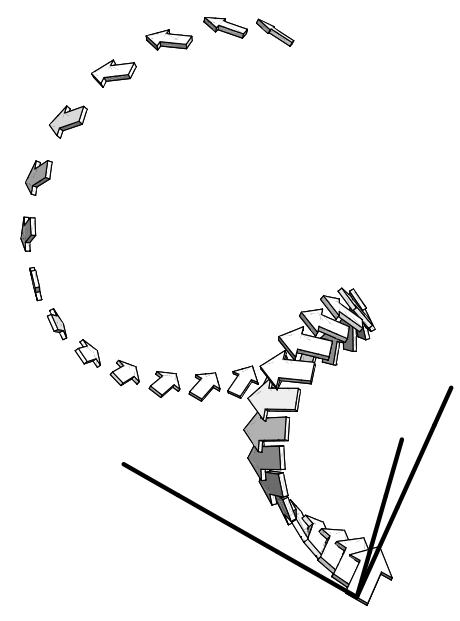

Fig. 5. A Brockett motions of the $\overline{\mathrm{IB}^{0}}$ type based on a pure translation. The parameters for this motion are $v_{1}=1, v_{2}=2, \omega_{1}=3, \omega_{2}=4$ and $z=-1$.

A general element of $\mathcal{L}_{1}$ can be written as a $4 \times 4$ matrix as,

$$
Z_{1}=\left(\begin{array}{cccc}
0 & 0 & \omega_{2} & v_{1} \\
0 & 0 & -\omega_{1} & v_{2} \\
-\omega_{2} & \omega_{1} & 0 & 0 \\
0 & 0 & 0 & 0
\end{array}\right)
$$

Notice that $\mathcal{L}_{0}$ is subgroup of cylindrical displacements hence $e^{t Z_{0}}$ is a uniform screw motion, or a uniform translation along the axis of the cylinder. In the case that $e^{t Z_{0}}$ is a uniform translation, we can write explicitly,

$$
e^{t Z_{0}}=\left(\begin{array}{cccc}
1 & 0 & 0 & 0 \\
0 & 1 & 0 & 0 \\
0 & 0 & 1 & t z \\
0 & 0 & 0 & 0
\end{array}\right)
$$

and hence the stationary control is,

$$
U(t)=e^{-t Z_{0}} Z_{1} e^{t Z_{0}}=\left(\begin{array}{cccc}
0 & 0 & \omega_{2} & v_{1}+t \omega_{2} z \\
0 & 0 & -\omega_{1} & v_{2}-t \omega_{1} z \\
-\omega_{2} & \omega_{1} & 0 & 0 \\
0 & 0 & 0 & 0
\end{array}\right)
$$

An example of this type of motion is illustrated in Fig. 5. These motions are not generated by curves, so they are illustrated by giving a sequence positions for a rigid body following the motion.

When $e^{t Z_{0}}$ is a uniform screw motion, with pitch $p$ we have,

$$
e^{t Z_{0}}=\left(\begin{array}{cccc}
\cos \omega t & -\sin \omega t & 0 & 0 \\
\sin \omega t & \cos \omega t & 0 & 0 \\
0 & 0 & 1 & p \omega t \\
0 & 0 & 0 & 1
\end{array}\right)
$$

and the corresponding stationary control is,

$$
U(t)=e^{-t Z_{0}} Z_{1} e^{t Z_{0}}=\left(\begin{array}{cccc}
0 & 0 & w_{2} & u_{1} \\
0 & 0 & -w_{1} & u_{2} \\
-w_{2} & w_{1} & 0 & 0 \\
0 & 0 & 0 & 0
\end{array}\right)
$$

where,

$$
\begin{aligned}
& w_{1}=\omega_{1} \cos \omega t+\omega_{2} \sin \omega t \\
& w_{2}=\omega_{2} \cos \omega t-\omega_{1} \sin \omega t \\
& u_{1}=\left(v_{1}+p \omega \omega_{2} t\right) \cos \omega t+\left(v_{2}-p \omega \omega_{1} t\right) \sin \omega t \\
& u_{2}=\left(v_{2}-p \omega \omega_{1} t\right) \cos \omega t-\left(v_{1}+p \omega \omega_{2} t\right) \sin \omega t
\end{aligned}
$$

Examples of these motions with varying values for the pitch $p$ of the screw motion they are based on are illustrated in Fig. 6.

In this case there is a choice for the bi-invariant form or objective function. Writing $\mathbf{z}_{1}=\omega_{1}\left(\begin{array}{l}\mathbf{i} \\ \mathbf{0}\end{array}\right)+\omega_{2}\left(\begin{array}{l}\mathbf{j} \\ \mathbf{0}\end{array}\right)+$ $v_{1}\left(\begin{array}{l}\mathbf{0} \\ \mathbf{i}\end{array}\right)+v_{2}\left(\begin{array}{l}\mathbf{0} \\ \mathbf{j}\end{array}\right)$ the performance measure here is given by,

$$
\mathbf{z}_{1}^{T} Q \mathbf{z}_{1}=2 \beta\left(\omega_{1}^{2}+\omega_{2}^{2}\right)+2 \alpha\left(\omega_{1} v_{1}+\omega_{2} v_{2}\right)
$$

The stationary motions do not depend on the values of $\alpha$ and $\beta$ but the integral of the objective function $\Phi$ from equation (15) will depend on these constants.

\section{CONCLUSIONS}

It was Wu et al [15] who probably first introduced the idea of Lie triple systems into robotics. The focus of their work was the design of serial and parallel manipulators.

The last two examples in sections V-C and V-D are substantially more complicated than the example in section $\mathrm{V}-\mathrm{A}$. This means that to use the motions found in these two sections for interpolation it would be necessary to solve an inverse kinematics problem, similar to the inverse kinematics problem familiar from serial robot manipulators.

However, the main interest in these motions is theoretical. It is rare to find explicitly solvable problems and moreover these solutions are important, naturally defined, features of the group of rigid-body displacements.

Finally, it is well-known that there is a close connection between the Lie triple systems of a Lie algebra, symmetric spaces and the involutions that can be defined on the Lie group, see [9] or [13], for example. It should be expected that involutions play a significant role in robotics and the theory of mechanisms, however this will not be pursued here. Except to note that the classification of the Lie triple systems carried out above will also give a classification of the possible involutions and that there appears to be a close connection between the 3 Lie triple systems whose closure is $s e(3)$ and the classical kinematic concepts of line symmetry, plane symmetry and point symmetry.

\section{APPENDIX A}

\section{A Brief Introduction to Screw TheOry.}

\section{A. Twists}

Elements of the Lie algebra se(3) are also known as twists. These vectors can be used to represent generalised velocities of a rigid-body. It is convenient to partition these 6-dimensional vectors as two 3-vectors; the angular $\boldsymbol{\omega}$, and linear $\mathbf{v}$, velocities of the rigid-body,

$$
\mathbf{s}=\left(\begin{array}{l}
\omega \\
\mathbf{v}
\end{array}\right)
$$



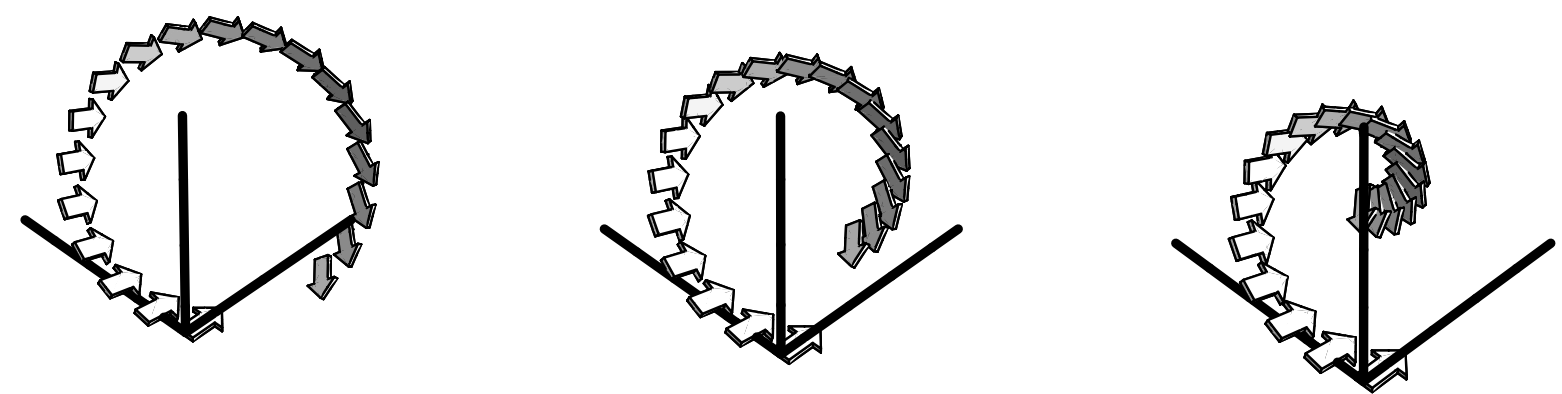

Fig. 6. Three general Brockett motions of the $\overline{\mathrm{IB}^{0}}$ type. The parameters are $\omega=1, v_{1}=-1, v_{2}=2, \omega_{1}=1, \omega_{2}=1$, for all three motions with, from left to right, $p=0, p=0.25$ and $p=0.5$.

The adjoint representation of the Lie algebra is given by $6 \times 6$ matrices partitioned as,

$$
\operatorname{ad}(\mathbf{s})=\left(\begin{array}{cc}
\Omega & 0 \\
V & \Omega
\end{array}\right)
$$

where $\Omega$ and $V$ are the $3 \times 3$ anti-symmetric matrices corresponding to $\boldsymbol{\omega}$ and $\mathbf{v}$ respectively. That is,

$$
V=\left(\begin{array}{ccc}
0 & -v_{z} & v_{y} \\
v_{z} & 0 & -v_{x} \\
-v_{y} & v_{x} & 0
\end{array}\right)
$$

so that $V \mathbf{x}=\mathbf{v} \times \mathbf{x}$ for arbitrary 3-vectors $\mathbf{x}$.

The Lie algebra structure on $s e(3)$ is given by the, so called, dual vector product,

$$
\left[\left(\begin{array}{c}
\boldsymbol{\omega}_{1} \\
\mathbf{v}_{1}
\end{array}\right),\left(\begin{array}{c}
\boldsymbol{\omega}_{2} \\
\mathbf{v}_{2}
\end{array}\right)\right]=\left(\begin{array}{c}
\boldsymbol{\omega}_{1} \times \boldsymbol{\omega}_{2} \\
\boldsymbol{\omega}_{1} \times \mathbf{v}_{2}+\mathbf{v}_{1} \times \boldsymbol{\omega}_{2}
\end{array}\right) .
$$

Elements of the dual to the Lie algebra, $s e(3)^{*}$ are often used to represent wrenches, combinations of forces and moments. However, they can also be used to represent momenta.

$$
\mathbf{p}=\left(\begin{array}{c}
\mathbf{m} \\
\mathbf{l}
\end{array}\right) \text {, }
$$

where $\mathbf{m}$ is the body's angular momentum and $\mathbf{l}$ is its linear momentum. In this way the evaluation of the momentum on the velocity is given by,

$$
\mathbf{p}^{T} \mathbf{s}=\mathbf{m} \cdot \boldsymbol{\omega}+\mathbf{l} \cdot \mathbf{v},
$$

which is twice the kinetic energy of the body. In this work no masses or inertias are considered so the elements of the conjugate vector $\mathbf{p}$ cannot be considered as a physical momentum.

Finally here, the bi-invariant forms on the Lie algebra can be found to be arbitrary linear combinations of a pair of basic forms,

$$
Q=\alpha Q_{0}+\beta Q_{\infty},
$$

where,

$$
Q_{0}=\left(\begin{array}{cc}
0 & I_{3} \\
I_{3} & 0
\end{array}\right), \quad \text { and } \quad Q_{\infty}=\left(\begin{array}{cc}
2 I_{3} & 0 \\
0 & 0
\end{array}\right) .
$$

To keep $Q$ invertible the constant $\alpha$ must not vanish, but the other constant $\beta$ can take any real value. An important invariant of the twists are their pitch which can be given in terms of these bi-invariant forms as,

$$
\text { pitch }=\frac{\mathbf{s}^{T} Q_{0} \mathbf{s}}{\mathbf{s}^{T} Q_{\infty} \mathbf{s}} .
$$

Pitch 0 twists, that is twists for which $\mathbf{s}^{T} Q_{0} \mathbf{s}=0$ are infinitesimal rotations. Non-zero twist for which both $\mathbf{s}^{T} Q_{0} \mathbf{s}=$ $\mathbf{s}^{T} Q_{\infty} \mathbf{s}=0$ are infinitesimal translations. The pitch of an infinitesimal translation is usually said to be infinite.

More details of this view of $s e(3)$ can be found in [10].

\section{B. Screw Systems}

A screw system is a vector subspace of $s e(3)$, that is a linear system of twists. These subspaces have been classified up to the action of the group $S E(3)$ of rigid displacements. This was first done in a heuristic way by Hunt and then a formal proof was given by Gibson and Hunt [5]. A cleaner version of the classification and proof was then given by Donelan and Gibson [6].

The Gibson-Hunt classification can be outlined as follows. The first thing to consider is the dimension of the system. An $n$-system, is an $n$ dimensional vector subspace of the six dimensional space $s e(3)$. Hence, $0<n \leq 6$. Next, we only need to consider 1,2 and 3-systems of screws; the 4 and 5 -systems are classified by their reciprocal 2 and 1-systems respectively. The reciprocal of a screw-system $\mathcal{S}$, is given by,

$$
\overline{\mathcal{S}}=\left\{\mathbf{s}: \mathbf{s}^{T} Q_{0} \mathbf{z}=0, \text { for all } \mathbf{z} \in \mathcal{S}\right\},
$$

where $Q_{0}$ is the symmetric matrix introduced in section A-A above.

The 1-systems are screws, that is a twist multiplied by an arbitrary non-zero factor. These are completely classified by their pitch, see equation (89). That is, two screws differ by a rigid-body displacement if and only if their pitches are equal. To classify 2 and 3 systems (and hence 4 and 5-systems), consider the family of quadric hypersurfaces in $\mathbb{P}^{5}$ determined 
by the $6 \times 6$ symmetric matrices,

$$
Q_{p}=\alpha Q_{0}+\beta Q_{\infty}
$$

where $Q_{0}$ and $Q_{\infty}$ are as described in equation (88). These quadrics are known as the pitch quadrics. In this geometric view a screw-system is a linear subspace of $\mathbb{P}^{5}$, that is a 2-system corresponds to a projective line, a 3-system to a projective plane. It might happen that the screw-system under consideration lies entirely in one of the pitch quadrics, this would be referred to as a II system of screws. Notice that in a II system (almost) all the screws will have the same pitch. The only exceptions have infinite pitch.

If the screws in a screw system have different pitches then the system is called a I system and it will intersect (almost) all the pitch quadrics. This partitions the screw-systems into two possible classes.

These classes can be further sub-divided by observing how they meet the pitch-quadric $Q_{\infty}$. This quadric is degenerate and forms a 2-plane which lies in all of the other pitch quadrics. The linear space corresponding to the screw-system under consideration might not meet $Q_{\infty}$, in this case we say that the system is an $A$ system. If the screw-system meets $Q_{\infty}$ in a point then we have a $B$ system and so forth.

Hence it is possible to find 2-systems of class IA, IB, IIA, IIB and IIC. The 3-systems fall into classes, IA, IB, IC,IIA, IIB, IIC and IID. The IID system is the unique 3 -system that consists of all of $Q_{\infty}$, that is the set of all infinite pitch twists.

In general each of these classes contains many screwsystems and these can be classified more finely. The intersection of the screw-systems with the pitch quadrics is a much studied object in classical Algebraic geometry-a pencil of quadrics. The final level of classification distinguishes screwsystems for which the projective type of this pencil of quadrics is different. In many cases there are continuous families of inequivalent screw-systems and these will be distinguished by one or more moduli. The simplest example here is the 1systems, there is a one-parameter family of equivalence classes of 1-systems these are distinguished by a single modulus the pitch.

In tables II and III above, the Gibson-Hunt class of the system $\mathcal{L}_{1}$ is referred to as its $\mathrm{GH}$ type. Notice that, the subalgebras of $s e(3)$ form screw systems and the Gibson-Hunt type of the subalgebras can be found quite simply. For more details of the classification see the references cited above and also, [10, chap. 8].

\section{ACKNOWLEDGMENT}

The author would like to thank R.W. Brockett for helpful comments on this work. The work has also been much improved following the comment of the anonymous reviewers.

\section{REFERENCES}

[1] V.I. Arnol'd, 1978, Mathematical Methods of Classical Mechanics, Springer, New York.

[2] R.L. Bishop, 1975, "There is more than one way to frame a curve", Am. Math. Monthly, 82:246-251.
[3] R. W. Brockett, 1999, "Explicitly solvable control problems with nonholonomic constraints". In Proceedings of the 38th Conference on Decision \& Control, Phoenix, Arizona, 1999.

[4] F. Bullo and A. D. Lewis, 2004, Geometric Control of Mechanical Systems, Springer, New York.

[5] C.G. Gibson and K.H. Hunt, 1990, "Geometry of screw systems", Mechanism and Machine Theory, 25:1-27.

[6] P.S. Donelan and C.G. Gibson, 1993, "On the hierarchy of screw systems", Acta Appl. Math., 32:267-296.

[7] N. Jacobson, 1949, "Lie and Jordan triple systems", American Journal of Mathematics, 71(1):149-170.

[8] V. Jurdjevic, 1997, Geometric Control Theory, Cambridge Studies in Advanced Mathematics (No. 52), Cambridge University Press.

[9] H. Z. Munthe-Kaas, G.R.W. Quispel, A. Zanna, 2013, "Symmetric spaces and Lie triple systems in numerical analysis of differential equations", arXiv: $1302.3387 \mathrm{v} 1$

[10] J.M. Selig, 2005, Geometric Fundamentals of Robotics. Springer Verlag, New York.

[11] J.M. Selig, 2013, "Characterisation of Frenet-Serret and Bishop Motions with applications to Needle Steering", Robotica, 31(6):981-992.

[12] J.M. Selig, 2014, "Some Rational Vehicle Motions", in Computational Kinematics: Proceedings of the 6th International Workshop on Computational Kinematics (CK2013), Mechanisms and Machine Science, Vol. 15, pp. 21-29. Editors Thomas, F. and Perez Gracia, A., Springer Verlag, Dordrecht.

[13] K. Spindler, 1994, "Cartan Algebras and Involutions", Proceedings of the American Mathematical Society, 121(2):323-333.

[14] K. Spindler, 2013, "Optimal Control on Lie Groups: Theory and Applications", WSEAS Transactions on Mathematics, 12(5):531-542.

[15] Y. Wu, G. Liu, H. Löwe, Z.X. Li, 2013, "Exponential Submanifolds: A New Kinematic Model For Mechanism Analysis and Synthesis", IEEE International Conference on Robotics and Automation (ICRA) Karlsruhe, Germany, pp. 4177-4182.

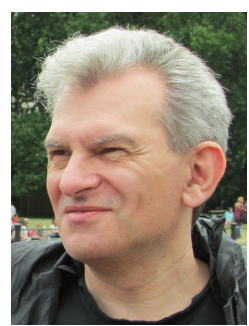

J.M. Selig graduated from the University of York, with a BSc in Physics in 1980. He went on to study in the Department of Applied Mathematics and Theoretical Physics at the University of Liverpool and was awarded a PhD in 1984. From 1984 to 1987 he was a postdoctoral research fellow in the design discipline of the Open University, studying robot gripping. He joined the Department of Electrical and Electronic Engineering at South Bank Polytechnic in 1987. In 1992 the Polytechnic became a University, and in 1999 he transferred to the School of Computing, Information Systems and Mathematics. In 2015, after another reorganisation, he transferred back to the School of Engineering after a time in the Faculty of Business. Jon's research interests can be summarised as the applications of modern geometry to problems in robotics. 